Research paper

\title{
Numerical analysis of a reciprocating active magnetic regenerator
}

\author{
Sergiu Lionte a, b, *, Carmen Vasile a, b, Monica Siroux ${ }^{\text {a, c }}$ \\ a The National Institute of Applied Sciences, INSA, 24 Bd. de la Victoire, 67084 Strasbourg, France \\ ${ }^{\mathrm{b}}$ LGeCo Laboratory EA 3938, 24 Bd. de la Victoire, 67084 Strasbourg, France \\ c ICUBE UMR 7357, 300 Bd. Sébastien Brant, 67412 Illkirch, France
}

\section{H I G H L I G H T S}

- We have developed a 2D model of an active magnetic regenerator.

- The MCE is included as a source term with data from experimental measurements.

- A validation of the model with experimental data is included.

- We analysed the temperature span, the cooling power, the COP and the pressure drop of the system.

\section{A R T I C L E I N F O}

\section{Article history:}

Received 15 July 2014

Accepted 21 October 2014

Available online 30 October 2014

\section{Keywords:}

Magnetic refrigeration

Active magnetic regenerator

Numerical simulation

\begin{abstract}
A B S T R A C T
A time-dependent, two-dimensional mathematical model of a configuration system for magnetic refrigeration has been developed, based on a reciprocating active magnetic regenerator operating at room temperature. The model's geometry is made of parallel plates of magnetocaloric material separated by microchannels. Through the microchannels, the flow of a heat transfer fluid has also been simulated. Water has been used as heat transfer fluid and as magnetocaloric material we have used the benchmark material gadolinium. The heat transfer inside the regenerator and the fluid flow are modelled separately and the magnetocaloric effect is taken into account by the inclusion of a variable source term in the energy equation.

The model simulates the steps of the active magnetic regenerative refrigeration cycle and evaluates the performance in terms of cooling load, COP, temperature span and pressure drop for the parallel-plate configuration. The model has been validated by comparing the numerical results with the results obtained from an experimental device made by a partner. This parametric study allows us to identify the most important characteristics that have a significant influence on the thermal behaviour of the active magnetic regenerator. Several simulation results are discussed and some optimal solutions are presented.
\end{abstract}

๑) 2014 Elsevier Ltd. All rights reserved.

\section{Background and introduction}

Almost all today's refrigeration is based on vapour-compression technology that was introduced more than 100 years ago. Now it is a mature, reliable and low-cost technology but is close to reach its technical boundaries and it uses refrigerants gas harmful for the environment. International pressure to phase down slowly but surely the use of HFCs as refrigerants has been mounting over the past 4 years. Late 2012, the European Commission proposed to implement a $79 \%$ reduction in the production and consumption of HFCs by 2030 , along with a ban on high-GWP refrigerants. More

\footnotetext{
* Corresponding author. The National Institute of Applied Sciences, INSA, 24 Bd. de la Victoire, 67084 Strasbourg, France.

E-mail address: sergiu.lionte@insa-strasbourg.fr (S. Lionte).
}

than ever, alternative solutions should be implemented whenever alternatives already exist and the seeding up of technological development in other applications should also be achieved [1].

Room-temperature magnetic refrigeration completely eliminates the use of HFCs and could also reduce the direct energy consumption comparing to the classical refrigeration technology. Recently a series of magnetic refrigeration prototypes have been created and constructed witch have obtained a COP of $10-15$, when the traditional refrigeration COPs are between 2 and 4, as seen in Gschneider et al. [2].

Magnetic refrigeration is an emergent cooling technology that exploits the magnetocaloric effect (MCE), of different metals and alloys. This effect was discovered experimentally by Emil Wartburg in iron in 1881, but only later, Debye (1926) and Giauque (1927) proposed to use reversible temperature change in paramagnetic salts to obtain low temperatures by adiabatic demagnetisation. The most 
common magnetocaloric material is gadolinium. Due to its availability only in small quantities compared to the significant demand provoked by the market potential, research is oriented towards other materials with a giant MCE, such as materials based on Lantane-IronSilicium (LaFeSi) with their respective variations $\left(\mathrm{LaFe}_{(13-x)} \mathrm{Si}_{x}\right.$, $\mathrm{LaFeSiH}$, etc.) or materials based on manganese such as MnFePAs or MnFePSi. Further explanations can be found is Legait et al. [3].

One of the problems of existing MCMs is that the adiabatic temperature change is relatively small. $\Delta T_{\mathrm{ad}}$ of gadolinium in a $1 \mathrm{~T}$ magnetic field is around $3 \mathrm{~K}$. To achieve a temperature span comparable to conventional refrigeration, amplification is therefore required. This amplification is made by the use of an active magnetic regenerator (AMR) which was first use in 1984 by Barclay et al. [4].

Using an AMR, the MCM is able to work as a regenerative material as well as a refrigerant. As a result, the temperature span between the cold end and the hot end are increased. More than 40 prototypes have been tested in the past few years, according to Plaznik et al. [5].

Lately, an important number of AMR numerical models have been developed so far. There are some 1D models which assume that the fluid flow and the thermal conduction (if it is included) occur only in the downstream fluid direction, as in the model of Tagliafico et al. [6], Risser et al. [7], Vuarnoz et al. [8], Roudaut et al. [9], and Tusek et al. [10]. In general, 1D models neglect the intraparticle thermal conduction and require the application of a heat transfer coefficient between the fluid and the solid matrix. 2D models of the AMR have been developed by Petersen et al. [11], Nielsen et al. [12] and Oliveira et al. [13]. They took into account the 2D velocity field, which is established in the direction of the fluid flow. In general, they also considered the thermal conduction in 2D (perpendicular and parallel to the fluid flow). Equations for the fluid and the solid matrix in 2D models are not directly coupled through the heat-transfer coefficient, but are connected through an additional boundary condition that defines the thermal contact between the fluid and the solid. The first attempt of the 3D AMR model is presented by Bouchard et al. [14]. In this model the solid magnetic material and the regeneration fluid are modelled separately and some special numerical schemes are used to avoid unrealistic computation time and memory requirements.
Most of these models use the magnetocaloric effect modelled via mean field theory (MFT) from the WDS model (Weiss mean field model, Debye model for lattice contribution and Sommerfeld model for electronic contribution) which is a theoretical wellknown model to implement the magnetocaloric effect.

The results of these models show differences to experimental data [15]. The differences are mainly caused by the heat transfer correlations, the simplified geometry and the use of the WDS model to simulate the MCE.

\section{Modelling the active magnetic regenerator}

In this paper we present a dynamic 2D model for the description of the transient flow and temperature fields in a regenerator with a geometry based on parallel plates of magnetocaloric material. The model uses the two-dimensional Navier-Stokes equations for the fluid flow between the solid plates which are alternatively heated and cooled during magnetization and demagnetization. The magnetocaloric effect is inserted as a source term and is based on the experimental data, as detailed in Section 2.3. In the end we explained the adopted numerical solution and the results.

\subsection{Regenerator design and parameters}

The geometry is based on a regenerator made of 31 parallel plates arranged in a stack, with a total height of $23.7 \mathrm{~mm}$, according to Fig. 1. In order to avoid very long computational times, based on symmetry, we simulated the solid domain with the width of a half of the plate's width and the fluid domain with the width of a half of a microchannel's width. We have two heat exchangers placed on both sides. The gaps between the regenerator and both heat exchangers are constructed in order to ensure that the heat transfer to and from the regenerator only occurs through the fluid and they are considered thermally insulated (Fig. 2).

The correlations used for the fluid displacement and the magnetic field disposal use the following time steps:

- Fluid displacement: $t_{\mathrm{stab}}=0.1 \mathrm{~s}$ and $t_{\text {cold blow }}=t_{\text {hot blow }}=0.2 \mathrm{~s}$;

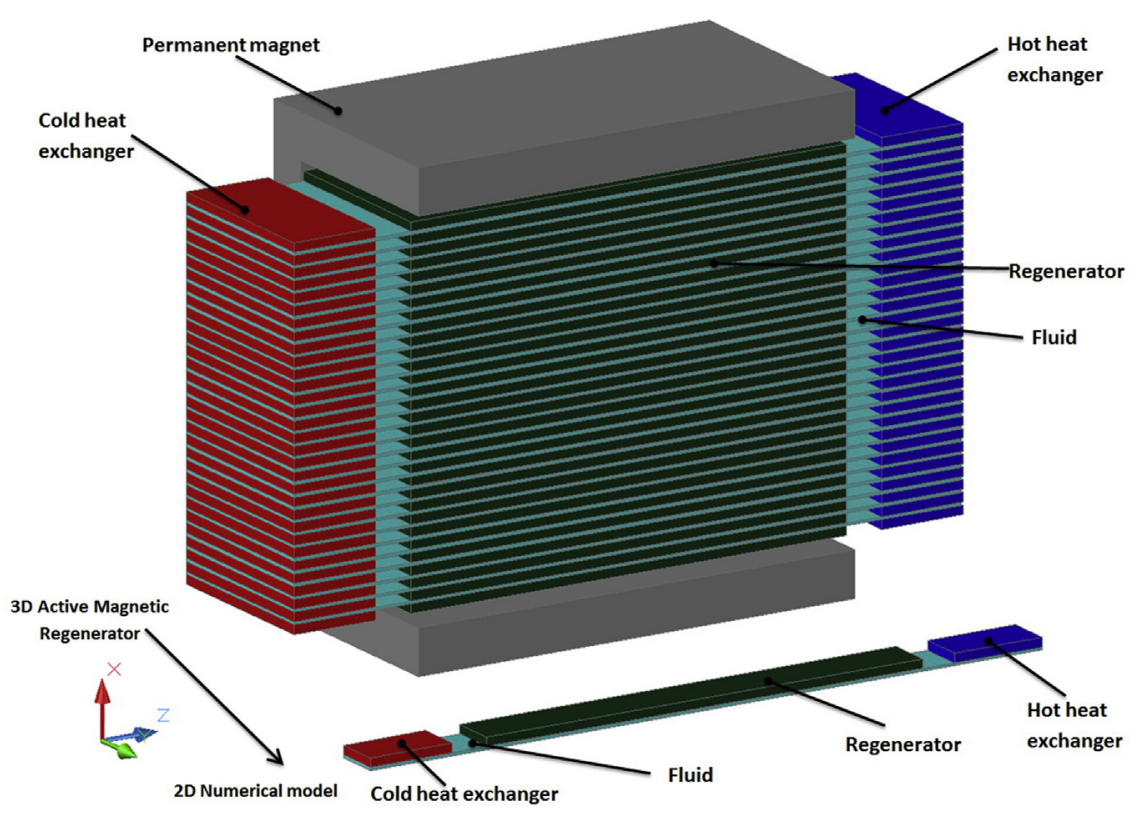

Fig. 1. The regenerator geometry including solid and fluid domains. 


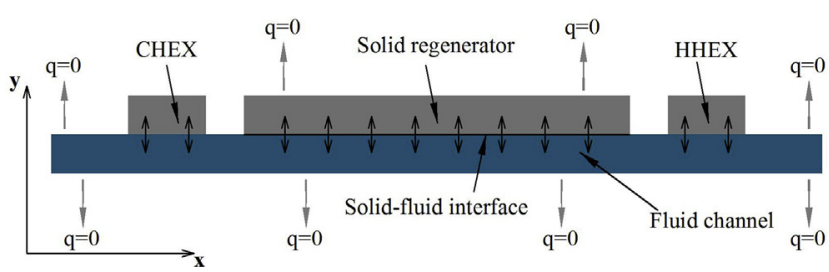

Fig. 2. The numerical model boundary conditions.

- Magnetic field disposal: $t_{\mathrm{mag}}=t_{\mathrm{demag}}=0.1 \mathrm{~s}$ and $t_{H \max }=t_{H \min }=0.4 \mathrm{~s}$.

Table 1 lists the geometrical and functioning parameters used in this study. The magnetocaloric material used for all the calculations is gadolinium and the heat transfer fluid is water. The magnetic field change is simulated from 0 to $1.1 \mathrm{~T}$ which can be obtained with permanent magnets. In our study we have also used temperature dependent physical parameters for gadolinium and water such as specific heat capacity $\left(c_{p}\right)$, thermal conductivity $(k)$, density $(\rho)$ and adiabatic temperature change $\left(\Delta T_{\mathrm{ad}}\right)$.

The gadolinium heat capacity and adiabatic temperature change are presented in paragraph 2.3.

\subsection{Governing equations and boundary conditions}

The model takes into consideration the fluid flow, the MCE and the heat transfer between the regenerator, the fluid and the heat exchangers. Based on these physics, the following assumptions are made:

1. The regenerator is perfectly insulated so that no heat losses occur from the solid or fluid to the surroundings;

2. The solid has a uniform density and the eddy currents and hysteresis losses are neglected;

3. The fluid is considered to be incompressible, and its mean velocity remains constant during the cold and hot blow periods and the fluid flow is fully developed and parallel to the longitudinal axis;

4. The solid and fluid are assumed to be in perfect thermal contact.

The velocity distribution is determined by solving the Navier-Stokes momentum and continuity equation for an incompressible fluid.

$\rho_{f} \cdot\left(\frac{\partial u}{\partial t}+(u \cdot \nabla) \mathrm{u}\right)-\mu_{f} \nabla^{2} u+\nabla p=0$

$\nabla \cdot \mathrm{u}=0$

To determine the temperature distribution in the AMR, the coupled heat transfer equations for the solid and the fluid domains

Table 1

Geometric and cycle parameters.

\begin{tabular}{lll}
\hline Parameter & Unit & Value \\
\hline$l$ & $\mathrm{~m}$ & $100 \times 10^{-3}$ \\
$w$ & $\mathrm{~m}$ & $20 \times 10^{-3}$ \\
$h$ & $\mathrm{~m}$ & $23.7 \times 10^{-3}$ \\
$\delta_{s}$ & $\mathrm{~m}$ & $0.6 \times 10^{-3}$ \\
$\delta_{f}$ & $\mathrm{~m}$ & $0.17 \times 10^{-3}$ \\
$\varepsilon$ & - & 0.215 \\
$\mathrm{fq}$ & $\mathrm{s}^{-1}$ & $0.3-2.0$ \\
$H_{\max }$ & $\mathrm{T}$ & 1.1 \\
$u$ & $\mathrm{M} \mathrm{s}^{-1}$ & $0.025-0.15$ \\
\hline
\end{tabular}

are solved. For the solid domains the temperature distribution is determined by the heat transfer equation with the MCE as the source term.

$\rho_{s} \cdot c_{p, s} \frac{\partial T_{s}}{\partial t}+\nabla \cdot\left(-k_{s} \nabla T_{s}\right)=\dot{Q}_{\mathrm{MCE}}+\dot{Q}_{\mathrm{HT}}$

The temperature distribution in the fluid is determined by the heat transfer equation for an incompressible fluid with convective terms.

$\rho_{f} \cdot c_{p, f}\left(\frac{\partial T_{f}}{\partial t}+(u \cdot \nabla) T_{f}\right)+\nabla \cdot\left(-k_{f} \nabla T_{f}\right)=-\dot{Q}_{\mathrm{HT}}$

The velocity distribution previously determined from Equations (1) and (2) is used as an input value for Equation (4) to determine the convective heat transfer. The heat transfer Equations ( 3 ) and (4) are linked by the heat transfer term $\dot{Q}_{H T}$.The source term in Equation (3) is the MCE flux defined as in Equation (5):

$\dot{Q}_{\mathrm{MCE}}=\rho_{\mathrm{s}} \cdot c_{p, s}(H, T) \cdot \frac{\Delta T_{\mathrm{ad}}(H, T)}{d t}$

The $c_{p, s}(H, T)$ and $\Delta T_{\mathrm{ad}}(H, T)$ are the solid material's thermophysical properties that depends on the magnetic field intensity and the material's initial temperature. These values are taken from experimental data, as shown in paragraph 2.3.

The solid and fluid are assumed to be in perfect thermal contact, driving the flux continuity, with the following boundary condition:

$k_{f} \frac{\partial T_{f}}{\partial y}=k_{s} \frac{\partial T_{s}}{\partial y}$

The solid and the fluid are initially at constant temperature. Boundary conditions are Neumann type at the exterior walls and Dirichlet type at the fluid entrance. For the solid-fluid interface we have a "no slip wall" condition.

There are changing boundary conditions at both ends of the regenerator depending on the movement direction. At the cold blow the temperature of the fluid is considered to be $T_{f}$ $(x=0, t)=T_{\text {cold }}$ and at the hot blow the temperature of the fluid is considered to be $T_{f}(x=L, t)=T_{\text {hot }}$ where $L$ is the regenerator's length.

\subsection{Magnetocaloric properties of gadolinium}

In order to obtain a more realistic AMR simulation, for the implementation of the magnetocaloric effect we have used a semiexperimental method by interpolation of measured data, taken from Risser's thesis [16]. The heat capacity and adiabatic temperature change for gadolinium are shown in Fig. 3.

The model has been solved using the discretization with the weak formulation of the Finite Elements Method which solves the PDEs in integral form using the commercial software COMSOL Multiphysics. In order to implement the fluid flow along the channel, a moving mesh has been implemented along the $x$ direction for the fluid domain. A structural grid topology has been used with 2100 domain elements and 760 boundary elements. The mesh is fine enough in order to count both for the fluid flow and for the heat transfer.

\subsection{Experimental data}

In order to validate the results obtained by the numerical model we have used the experimental data provided by our project partner, Cooltech Applications and published in Risser's thesis [16]. 

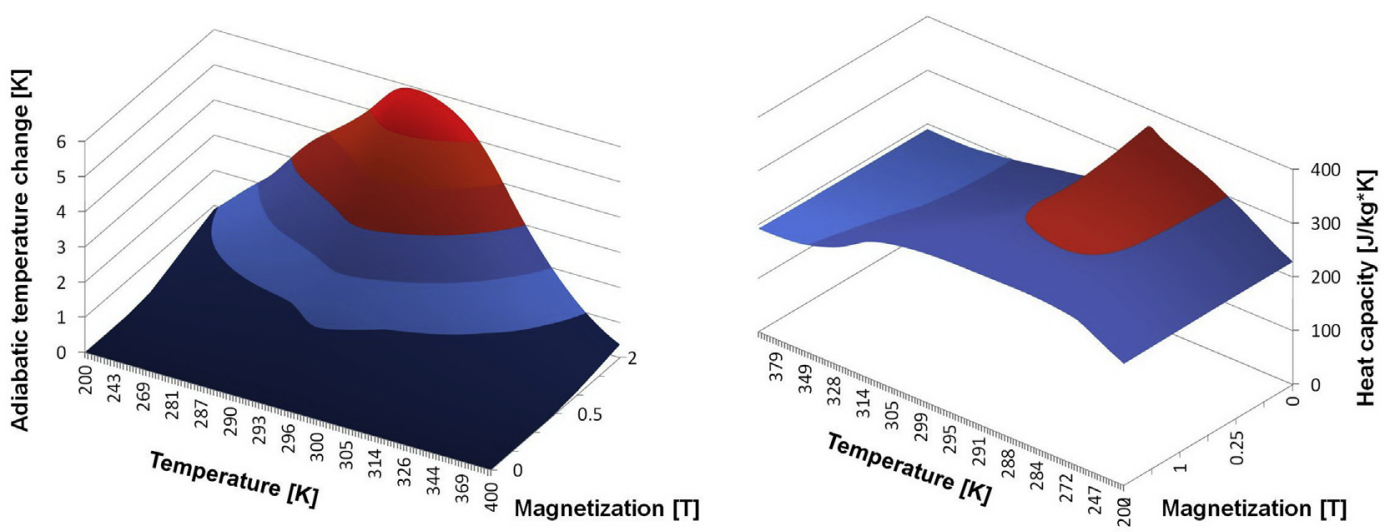

Fig. 3. Gadolinium magnetocaloric properties.

The AMR test device consists of a reciprocating system made from a fixed permanent magnet and a mobile regenerator. This prototype is called BIP and it is built by Cooltech Applications in Holtzheim, France. The regenerator is made of a stack of 31 gadolinium parallelled plates with a length of $100 \mathrm{~mm}$ and a thickness of $0.6 \mathrm{~mm}$. The microchannels are $0.17 \mathrm{~mm}$ thick. The prototype uses a magnetic field intensity of $1.1 \mathrm{~T}$, a frequency of $0.50 \mathrm{~Hz}$ and a fluid velocity of $0.03 \mathrm{~m} / \mathrm{s}$.

\section{Results and discussions}

In this section we will present the basic initial results in graphical form, i.e. the time evolution of the temperature gradient in the solid, the heat exchangers temperature evolution of the AMR for numerical simulation and the experimental device, the $2 \mathrm{D}$ velocity field for one microchannel and the 2D temperature distribution over the AMR at the characteristics phases of the cycle. Furthermore we will analyse the impact of the basic operational parameters.

\subsection{Initial results}

The AMR numerical model presented was designed to simulate the Active Magnetic Regenerative Refrigeration (AMRR) magnetic cycle. After a certain number of cycles (depending on the operating conditions) a steady-state is achieved. This happens when the fluid temperature at the exit of the AMR in two successive cycles does not change by more than $0.01{ }^{\circ} \mathrm{C}$. During steady-state operation a

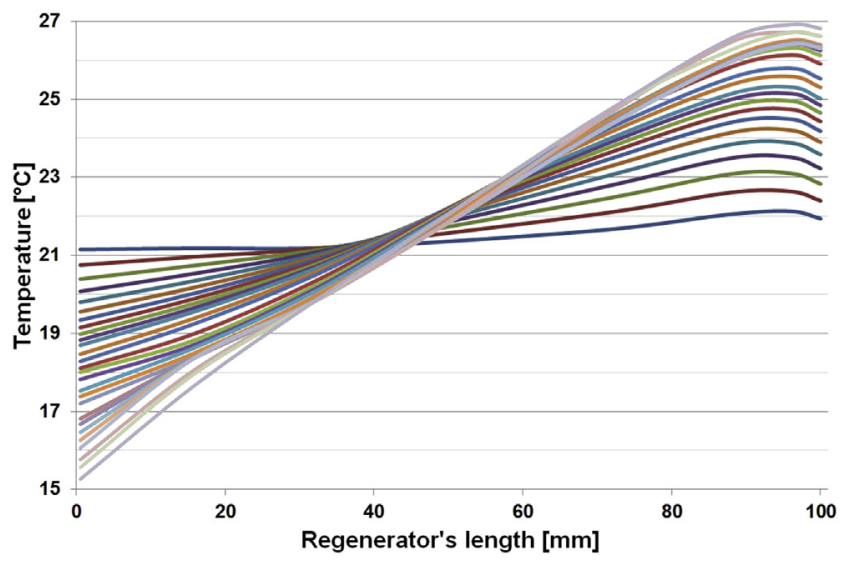

Fig. 4. Time evolution of the temperature gradient in the solid operating with no-load. temperature profile is formed along the length of the regenerator due to the reciprocating fluid flow. Thus, the cold and hot heat exchangers are at different temperatures. Fig. 4 shows the time evolution of the temperature gradient in the solid when the regenerator is working in no-load conditions. Initially, the solid is at the constant initial temperature of $21.30{ }^{\circ} \mathrm{C}$. Then the thermal gradient increases and it approaches a curved profile with a temperature difference and a profile that depends on the operation parameters. The simulation summed a number of 2000 time steps but in Fig. 4 we represented just 20 time steps, in order to see the time evolution of the temperature.

In Fig. 5 we can observe the transient evolution of the temperatures in the AMR working at $0.5 \mathrm{~Hz}$ with a fluid velocity of $0.03 \mathrm{~m} / \mathrm{s}$ and the temperature obtained by the experimental device. The simulation was performed over $200 \mathrm{~s}$, enough time for the steadystate to be achieved. The slow convergence towards the steadystate results in the long computational time of about $20 \mathrm{~h}$ of numerical simulation required to complete all $200 \mathrm{~s}$. The upper part of the figure is represented by the temperature evolution in the HHEX, followed by the temperature evolution in the CHEX in the lower part of the picture.

After $50 \mathrm{~s}$, in the numerical simulation, the regenerator enters progressively into the steady-state condition. In this phase, the temperature difference from one cycle to another doesn't change so much and the temperature profiles are almost linear. This is clearly illustrated by the fact that the change in the exchanger's temperatures is $5.6^{\circ} \mathrm{C}$ for the first $50 \mathrm{~s}$, while for the last $150 \mathrm{~s}$ the temperature increase is only $0.3^{\circ} \mathrm{C}$. The final temperature difference for

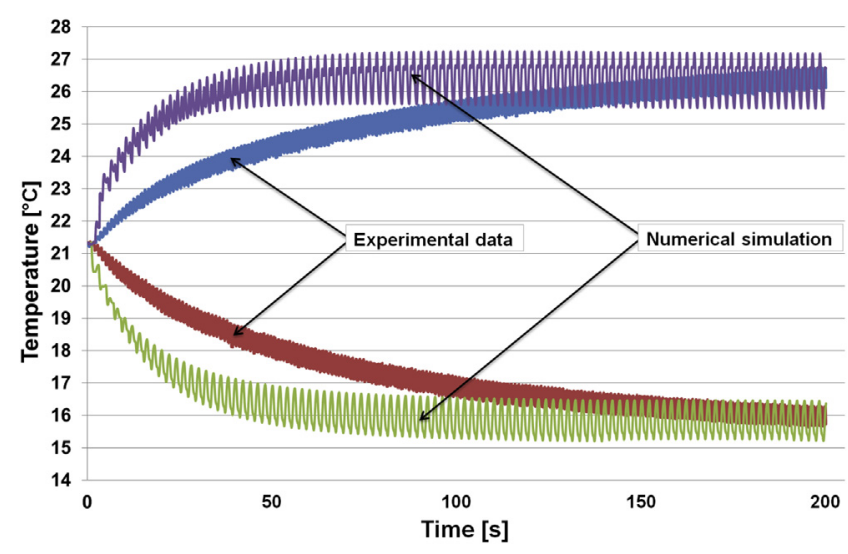

Fig. 5. The transient evolution of the representative temperatures in the AMR. 


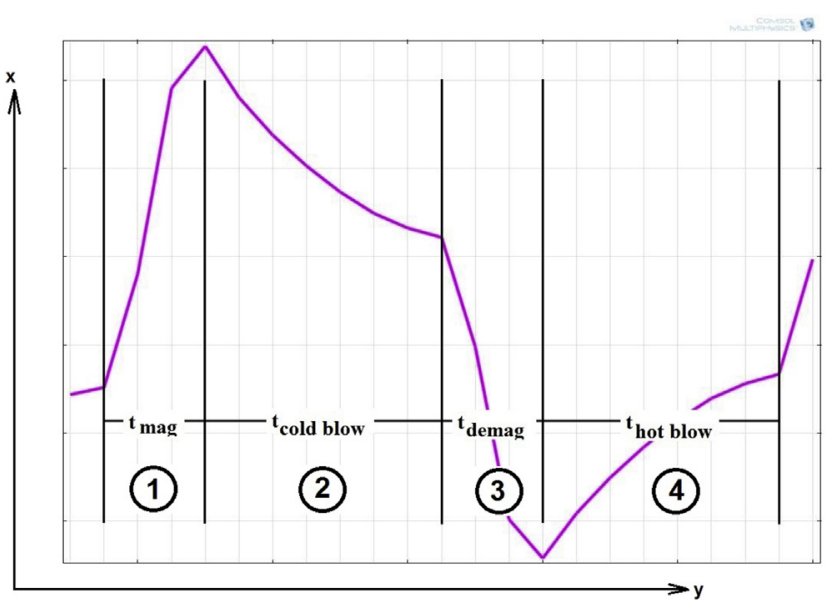

Fig. 6. Zoom on the time evolution of the temperature gradient in the solid part of AMR.

the numerical simulation is $11.85^{\circ} \mathrm{C}$ as for the experimental device is $11.12{ }^{\circ} \mathrm{C}$. The experimental device enters slower into the steadystate condition as we can observe from the temperatures profile which is sharper in the beginning of the temperature evolution. This is due mainly to the heat loses occurred to the environment.
The numerical model, due to the adiabatic conditions, has a perfect temperature evolution during one cycle, which allows us to observe all the four magneto-thermic phases, as detailed in Fig. 6.

Fig. 6 shows the form of the temperature evolution in the regenerator, during one cycle, divided into 4 characteristics phases. The temperature evolution is linked to the correlation between the fluid displacement and the magnetic field disposal.

In the magnetization process the regenerator's temperature increases due to the magnetocaloric effect. Afterwards, in the cold blow period, the fluid flows through the regenerator, taking a part of the heat and the regenerator's temperature is reduced. In the demagnetization period, the temperature in the solid is reduced even more when the magnetic field is removed. In the hot blow period, the solid is regenerated from the fluid, and the temperature is increased.

This process can be observed also in Fig. 7 where the system's temperatures are represented in a 2D scheme with the regenerator in the middle, the CHEX in the left part of the figure, the HHEX in the right part and the fluid channel which connects all three solid domains.

\subsection{Regenerator performance}

In Fig. 8 the maximum temperature span achieved by the model is presented as a function of time, showing the temperature

a) Magnetization

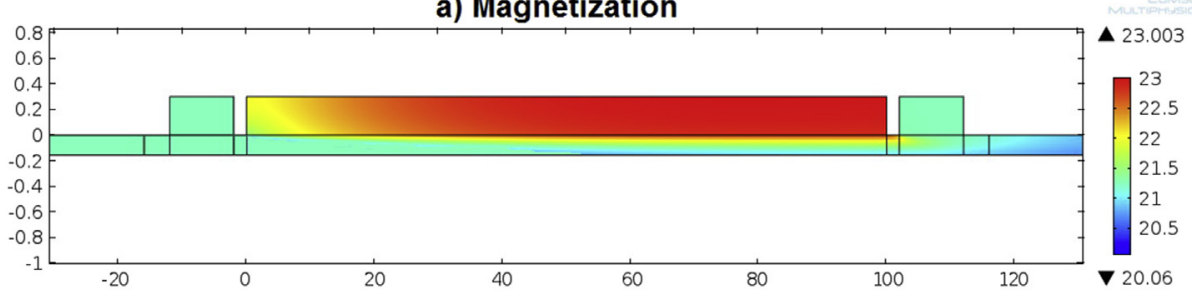

b) Cold blow

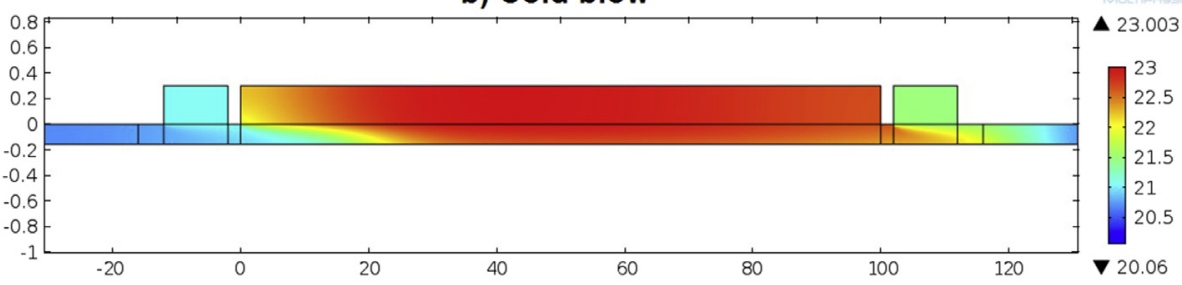

c) Demagnetization

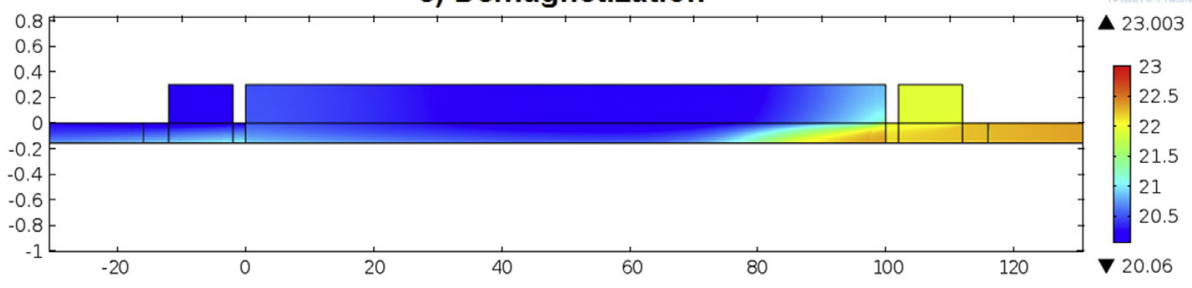

d) Hot blow

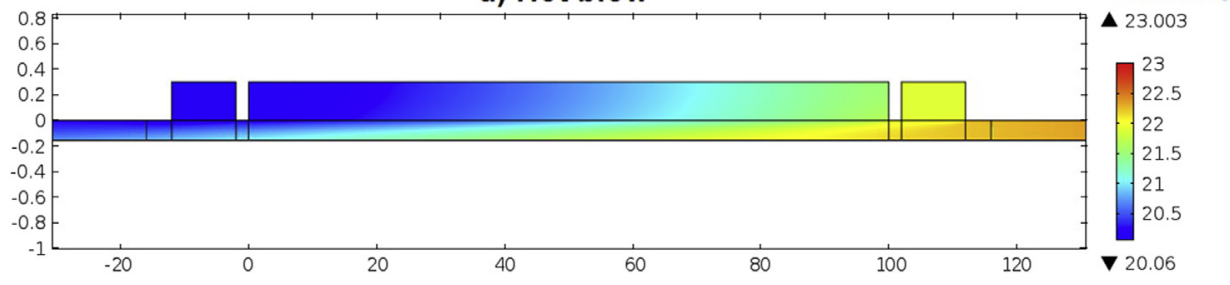

Fig. 7. The $2 \mathrm{D}$ temperature distribution of the system at the main phases of the cycle. 


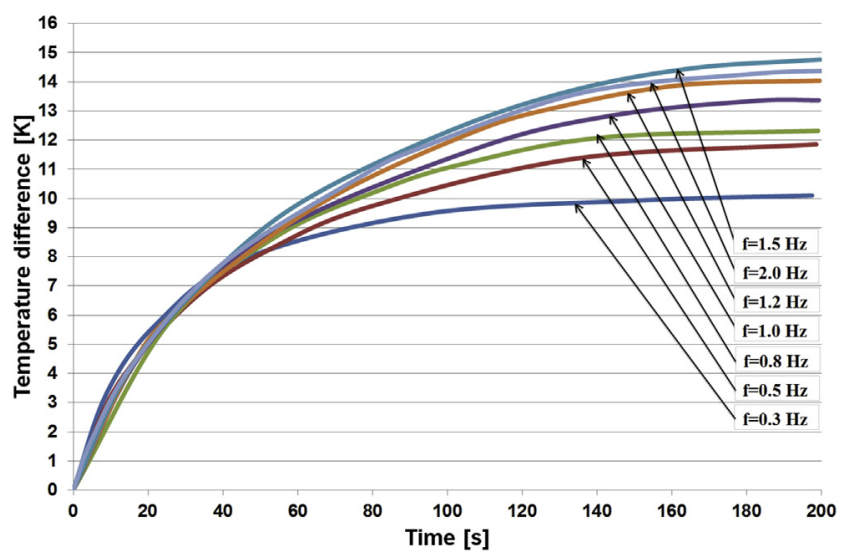

Fig. 8. Time evolution of the temperature span at no-cooling load for different operating frequencies.

difference between the CHEX and the HHEX. The simulations were carried out for $200 \mathrm{~s}$, in order to achieve steady-state condition.

The greatest temperature difference, of $14.5{ }^{\circ} \mathrm{C}$, is obtained when the system is working at a frequency of $1.5 \mathrm{~Hz}$ and the smallest temperature difference, of $10.1{ }^{\circ} \mathrm{C}$, at $0.3 \mathrm{~Hz}$. For all the studied frequencies the temperature span increases smoothly on the profile line. This means that the velocity is correlated with the frequency and the system is working properly. Also the temperature difference between the heat exchangers increases with the increase of the frequency from 0.3 to $1.5 \mathrm{~Hz}$. Above, at the frequency of $2.0 \mathrm{~Hz}$, the temperature difference is $13.6^{\circ} \mathrm{C}$, which is inferior to the one obtained at $1.5 \mathrm{~Hz}$. This is due to the fact that the system is working too fast and the fluid cannot extract all the heat properly from the solid.

The temperature difference between the exchangers increases with increasing frequency until $1.5 \mathrm{~Hz}$. This happens because once with the frequency increase, the period of the movement decreases and along with this, the distance travelled by the fluid inside the regenerator becomes smaller. Therefore, it is created a more significant thermal gradient inside the regenerator which leads to an increase in the temperature difference between the regenerator's ends and also between the regenerator's heat exchangers.
In order to quantify the operation of the regenerator and its performance the ratio of displaced fluid, $V^{*}$ vas recently introduced by Vuarnoz et al. [8]. This represents the ratio of the fluid mass displaced during the flow period with the respect to the mass of the fluid inside the AMR during one half-cycle and according to velocity scheme.

$V^{*}=\frac{\dot{M}_{f}}{f q \cdot \rho_{f} \cdot V_{\mathrm{AMR}} \cdot \varepsilon}$

where $\varepsilon$ is the porosity and defined as the ratio between the fluid volume inside the regenerator and the total volume of the regenerator.

The cooling power is calculated as:

$\dot{Q}_{\text {cold }}=\frac{1}{p} \int_{0}^{t_{\text {blow }}} \dot{M} \cdot c_{p . f}\left(T_{\text {cold }}-T_{f}(t)\right) \mathrm{d} t$

The amount of heat rejected from the system is obtained by the following equation:

$\dot{Q}_{\text {hot }}=\frac{1}{p} \int_{0}^{t_{\text {blow }}} \dot{M} \cdot c_{p, f}\left(T_{f}-T_{\text {hot }}(t)\right) \mathrm{d} t$

The COP is obtained as follows:

$\mathrm{COP}=\frac{Q_{\text {cold }}}{W_{\text {mag }}+W_{\text {pump }}}=\frac{Q_{\text {cold }}}{Q_{\text {hot }}-Q_{\text {cold }}+W_{\text {pump }}}$

The work of the pump, $W_{\text {pump }}$ is calculated in Section 3.2, in Equation (11).

Fig. 9 shows the cooling power as a function of temperature difference between the heat exchangers for a fluid velocity of $0.03 \mathrm{~m} / \mathrm{s}$ and different rates of displaced fluid, corresponding to the system frequencies. The best performance in our cases is obtained for a value of $V^{*}$ of 0.18 , corresponding of a system's frequency of $0.50 \mathrm{~Hz}$. In all seven cases, the cooling power profile shows maximum values in the temperature difference range of $0.5-4{ }^{\circ} \mathrm{C}$ after which the performance decrease with the increase of the temperature difference between the heat exchangers. We can observe in the figure that the performance of the system

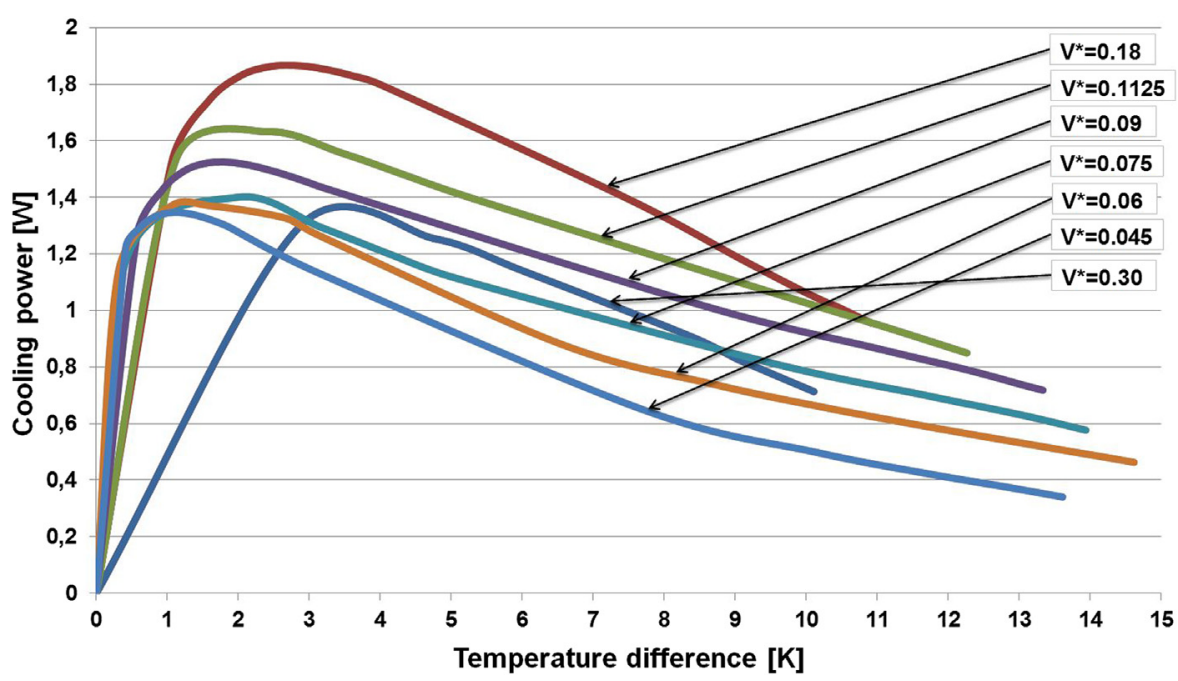

Fig. 9. The cooling power, as a function of temperature difference for different rates of displaced fluid for one single plate of gadolinium. 


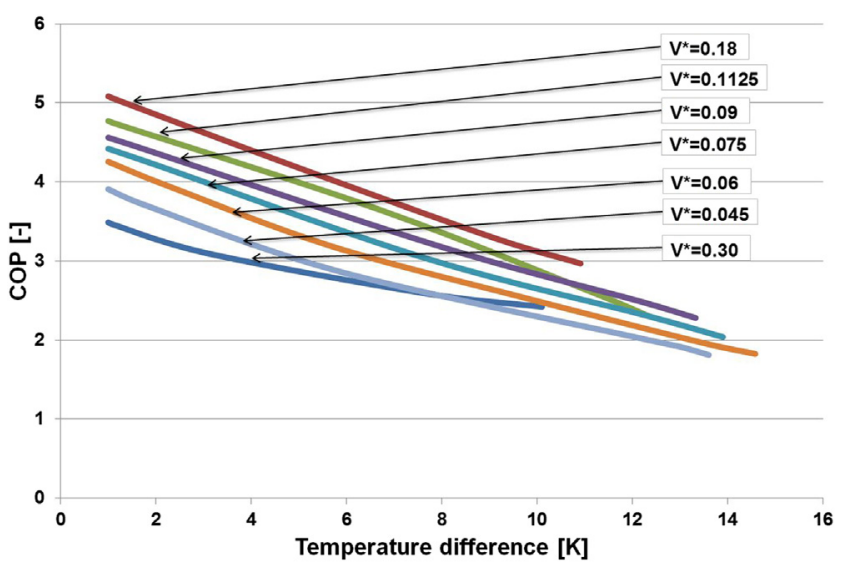

Fig. 10. The coefficient of performance, in no-load conditions, as a function of temperature difference for different rates of displaced fluid for one single plate of gadolinium.

decrease with the decrease of the ratio of displaced fluid in the range of 0.18 to 0.045 . The case where $V^{*}$ is 0.3 (frequency of $0.3 \mathrm{~Hz}$ ) does not follow the same pattern because the frequency is too low comparing to the fluid velocity and the system is not working properly. All the studied cases representation lines stop at different temperature difference which is the maximum temperature difference that can be achieved for each configuration of the working parameters of the system.

Fig. 10 shows the coefficient of performance as a function of the temperature span between the system's heat exchangers. The best performance is obtained when the system is working with a ratio of displaced fluid of 0.18 , corresponding to a frequency of $0.50 \mathrm{~Hz}$. The COP drops with the decrease of the $V^{*}$ for all but one of the studied cases. The COP for the case of $V^{*}$ equal to 0.3 is the lowest because of the frequency of 0.3 , which is too low and uncorrelated with the fluid velocity, as seen also in Fig. 9.

The pump work of the regenerator is expressed as the pressure drop multiplied by the volume flow, choosing a pump efficiency of 0.8 as in Kawanami et al. [17].

$W_{\text {pump }}=\frac{\dot{M}_{f} \cdot \Delta p}{\rho_{f} \cdot \eta}$

\subsection{Fluidics and pressure drop}

In Fig. 11 the 2D velocity profile for one microchannel is presented, for different values of mean fluid velocity. We can observe
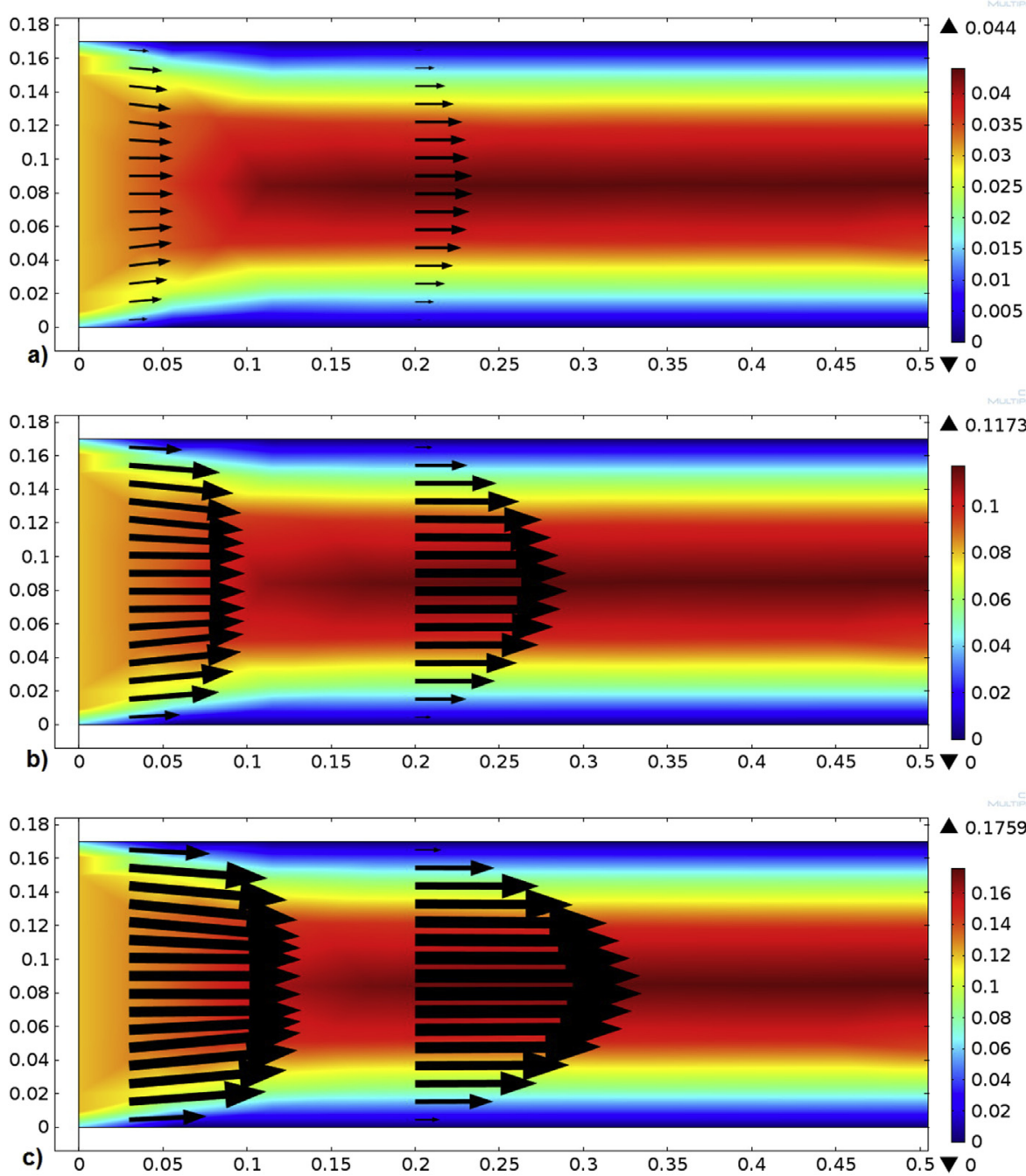

Fig. 11. The $2 \mathrm{D}$ velocity field for one microchannel and different medium velocity values: a) velocity $0.03 \mathrm{~m} / \mathrm{s}$ b) velocity $0.08 \mathrm{~m} / \mathrm{s}$ and c) velocity $0.12 \mathrm{~m} / \mathrm{s}$. 


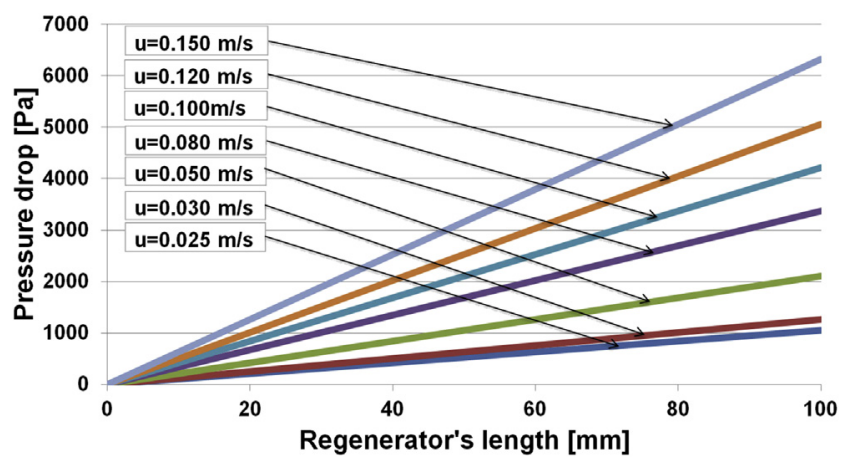

Fig. 12. The pressure drop of one microchannel for different rates of fluid velocity.

that the flow profile is completely established at $0.2 \mathrm{~mm}$, regardless the velocity value. This confirms the assumption that the fluid flow is fully developed over the total length of the microchannel.

For calculating the pressure drop and pumping power required we used the expression from Nielsen et al. model [12].

$\Delta p=\frac{96}{\operatorname{Re}} \cdot \rho_{f} \cdot \frac{L_{f}}{D_{H}} \cdot \frac{u^{2}}{2}$

with $\rho_{f}$ the fluid density, $L_{f}$ the microchannel length, $D_{H}$ the hydraulic diameter for rectangular microchannel and $u$ the mean fluid velocity.

In Fig. 12 we present the pressure drop for one microchannel as a function of fluid velocity. The Reynolds number varies from 9 (velocity $0.025 \mathrm{~m} / \mathrm{s}$ ) to 51 (velocity $0.15 \mathrm{~m} / \mathrm{s}$ ), so the flow is laminar. The low Reynolds number is due both to low velocity values and the low height of the microchannel $(0.17 \mathrm{~mm})$.

The pressure drop is directly influenced by the fluid velocity. The smallest pressure drop is found when the flow velocity is minimal $(0.025 \mathrm{~m} / \mathrm{s})$ and the greatest value of the pressure drop is obtained by the system working with the highest velocity $(0.150 \mathrm{~m} / \mathrm{s})$.

\section{Conclusions}

A 2D model of a reciprocating active magnetic regenerator has been developed and verified. The model is based on a configuration of parallel plates of gadolinium with water as heat transfer fluid. The magnetocaloric effect has been introduced as a source term and it is based on experimental data which enables the model to better reproduce the AMR behaviour. The model has been validated by comparing the numerical results with experimental data from a prototype.

In the first part of the paper we presented the initial results of the numerical model. We studied the evolution of the temperature gradient along the length of the regenerator, the temperature span of the heat exchangers and the 2D temperature distribution in the whole system (solid, fluid and heat exchangers).

In the second part we analysed the regenerator performance in terms of maximum temperature difference as a function of system frequency and the cooling power and coefficient of performance as a function of ration of displaced fluid.

The maximum temperature difference was obtained with the second maximum studied frequency. The maximum cooling power and COP were obtained with the system operating at the second maximum ratio of displaced fluid studied (which corresponds to the second minimum cycle frequency studied).
The third part was designated to fluidics and pressure drop. The $2 \mathrm{D}$ velocity field for multiple fluid velocities was presented and the pressure drop for one microchannel was analysed for different rates of fluid velocity.

The pressure drop is directly influenced by the fluid velocity, with bigger values for bigger velocities.

We concluded that although in terms of AMR quantitative studies such as the temperature difference, a bigger frequency is preferable, in terms of qualitative studies, better performance are obtained with lower frequencies (bigger ratio of displaced fluid). However, the frequency investigation also shows that it is very important to synchronize the value of the fluid velocity with the operating frequencies.

\section{Acknowledgements}

The authors would like to express their gratitude to the National Research Agency from France (Agence Nationale de Recherche), this study being supported by the project ANR-10-STOCK-E.

\section{Nomenclature}

MR magnetic refrigeration

AMRR active magnetic regenerative refrigeration

MCE magnetocaloric effect

HHEX hot heat exchanger

$S$

$T$

l

$w$

$h$

$\delta$

fq

$\rho$

$u$

$\mu$

$k$

$c_{p}$

$p$

pe

AMR active magnetic regenerator

MMC magnetocaloric material

CHEX cold heat exchanger

$H \quad$ magnetic field intensity, T

$A_{\mathrm{fs}} \quad$ fluid solid interface, $\mathrm{m}^{2}$

$\varepsilon \quad$ porosity, -

$t$ time, $s$

Qhot heat rejection, W

$Q_{\text {cold }}$ refrigeration capacity, $\mathrm{W}$

QMCE heat produced by EMC, W

$Q_{H T} \quad$ heat transferred by interface, $W$

$W_{\text {pump }}$ pumping power, $\mathrm{W}$

$W_{\text {mag }}$ magnetic power, $\mathrm{W}$

$M \quad$ mass flow rate, $\mathrm{kg} \mathrm{s}^{-1}$

$V^{*} \quad$ ratio of displaced fluid, -

$D_{H} \quad$ hydraulic diameter, $\mathrm{m}$

Re Reynolds number, -

$\eta \quad$ pump efficiency, -

\section{References}

[1] D. Coulomb, Int. J. Refrig. 36 (2013). Editorial.

[2] K. Gschneider Jr., V.K. Pecharsky, Rare earths and magnetic refrigeration, J. Rare Earths 24 (2006) 641-647. 
[3] U. Legait, F. Guillou, A. Kedous-Lebouc, V. Hardy, M. Almanza, An experimental comparison of four magnetocaloric regenerators using three different materials, Int. J. Refrig. 37 (2014) 147-155.

[4] J.A. Barclay, S. Steyert, Selection of regenerator geometry for magnetic refrigerator applications, in: Intersociety Cryogenic Symposium, 1984, pp. $1-12$.

[5] U. Plaznik, J. Tusek, A. Kitanovski, A. Poredos, Numerical and experimental analyses of different magnetic thermodynamic cycles with an active magnetic regenerator, Appl. Therm. Eng. 59 (2013) 52-59.

[6] G. Tagliafico, F. Scarpa, F. Canepa, A dynamic 1-D model for a reciprocating active magnetic regenerator; influence of the main working parameters, Int. J Refrig. 33 (2010) 286-293.

[7] M. Risser, C. Vasile, T. Engel, B. Keith, C. Muller, Numerical simulation of magnetocaloric system behaviour for an industrial application, Int. J. Refrig. 33 (2010) 973-981.

[8] D. Vuarnoz, T. Kawanami, Numerical analysis of a reciprocating active mag netic regenerator made of gadolinium wires, Appl. Therm. Eng. 35 (2011) 984-995.

[9] J. Roudaut, A. Kedous-Lebouc, J. Yonnet, C. Muller, Numerical analysis of an active magnetic regenerator, Int. J. Refrig. 34 (2011) 1797-1804.

[10] J. Tusek, A. Kitanovski, I. Prebil, A. Poredos, Dynamic operation of an active magnetic regenerator (AMR): numerical optimization of a packed-bed AMR Int. J. Refrig. 34 (2011) 1507-1517.
[11] T. Petersen, N. Pryds, A. Smith, J. Hattel, H. Schmidt, H. Knudsen, Twodimensional mathematical model of a reciprocating room-temperature active magnetic regenerator, Int. J. Refrig. (2007) 1-12.

[12] K.K. Nielsen, C.R.H. Bahl, A. Smith, N. Pryds, J. Hattel, A comprehensive parameter study of an active magnetic regenerator using a $2 \mathrm{D}$ numerical model, Int. J. Refrig. 33 (2010) 753-764.

[13] P. Oliveira, P. Trevizoli, J. Barbosa, T. Prata, A 2D hybrid model of the fluid flow and heat transfer in a reciprocating active magnetic regenerator, Int. J. Refrig. 35 (2012) 98-114.

[14] J. Bouchard, H. Nesreddine, N. Galanis, Model of a porous regenerator used for magnetic refrigeration at room temperature, Int. J. Heat Mass Transfer 50 (2008) 1223-1229.

[15] K. Nielsen, J. Tusek, K. Engelbrecht, Review on numerical modelling of active magnetic regenerators for room temperature applications, Int. J. Refrig. 34 (2011) 603-616.

[16] M. Risser, Modélisation Multiphysique et Multiconfigurationnelle d'Evaluation et d'Optimisation des Performances des Systèmes de Réfrigération Magnétocalorique (Ph.D. Thesis), University of Strasbourg, Strasbourg, France, 2011.

[17] T. Kawanami, S. Hirano, K. Fumoto, S. Hirasawa, Evaluation of fundamental performance on magnetocaloric cooling with active magnetic regenerator, Appl. Therm. Eng. 31 (2011) 1176-1183. 